\title{
Comparison of droplet digital PCR and conventional quantitative PCR for measuring EGFR gene mutation
}

\author{
BO ZHANG ${ }^{1}$, CHUN-WEI XU $^{1}$, YUN SHAO ${ }^{1}$, HUAI-TAO WANG $^{1}$, YONG-FANG WU ${ }^{1}$, YE-YING SONG ${ }^{1}$, \\ XIAO-BING LI ${ }^{1}$, ZHE ZHANG ${ }^{2}$, WEN-JING WANG ${ }^{2}$, LI-QIONG LI $^{2}$ and CONG-LI CAI ${ }^{2}$ \\ ${ }^{1}$ Department of Pathology, Affiliated Hospital of Academy of Military Medical Sciences, Beijing 100071; \\ ${ }^{2}$ Wuhan YZY Medical Science and Technology Co., Ltd., Wuhan, Hubei 430075, P.R. China
}

Received October 9, 2014; Accepted December 19, 2014

DOI: $10.3892 / \mathrm{etm} .2015 .2221$

\begin{abstract}
Early detection of epidermal growth factor receptor (EGFR) mutation, particularly EGFR T790M mutation, is of clinical significance. The aim of the present study was to compare the performances of amplification refractory mutation system-based quantitative polymerase chain reaction (ARMS-qPCR) and droplet digital polymerase chain reaction (ddPCR) approaches in the detection of EGFR mutation and explore the feasibility of using ddPCR in the detection of samples with low mutation rates. EGFR gene mutations in plasmid samples with different T790M mutation rates (0.1-5\%) and 10 clinical samples were detected using the ARMS-qPCR and ddPCR approaches. The results demonstrated that the ARMS-qPCR method stably detected the plasmid samples (6,000 copies) with 5 and $1 \%$ mutation rates, while the ddPCR approach reliably detected those with 5\% (398 copies), $1 \%$ (57 copies), $0.5 \%$ (24 copies) and $0.1 \%$ (average 6 copies) mutation rates. For the 10 clinical samples, the results for nine samples by the ARMS-qPCR and ddPCR methods were consistent; however, the sample N006, indicated to be EGFR wild-type by ARMS-qPCR, was revealed to have a clear EGFR T790M mutation with seven copies of mutant alleles
\end{abstract}

Correspondence to: Professor Bo Zhang, Department of Pathology, Affiliated Hospital of Academy of Military Medical Sciences, 8 Dong Da Street, Fengtai, Beijing 100071, P.R. China

E-mail: zenwo@qq.com

Dr Cong-Li Cai, Wuhan YZY Medical Science and Technology Co., Ltd., Biolake Building C2-1, 666 Gaoxin Road, Wuhan, Hubei 430075, P.R. China

E-mail: caicongli@yzybio.com

Abbreviations: EGFR, epidermal growth factor receptor; NSCLC, non-small cell lung cancer; TKIs, tyrosine kinase inhibitors; ARMS-qPCR, amplification refractory mutation system-based quantitative polymerase chain reaction; ddPCR, droplet digital polymerase chain reaction

Key words: epidermal growth factor receptor gene mutation, non-small cell lung cancer, droplet digital polymerase chain reaction, quantitative polymerase chain reaction in a background of 6,000 wild-type copies using ddPCR technology. This study demonstrates the feasibility of applying the ddPCR system to detect EGFR mutation and identified the advantage of ddPCR in the detection of samples with a low EGFR mutation abundance, particularly the secondary EGFR T790M resistance mutation, which enables early diagnosis before acquired resistance to tyrosine kinase inhibitors becomes clinically detectable.

\section{Introduction}

Lung cancer is the leading cause of cancer mortality and non-small cell lung cancer (NSCLC) accounts for $\sim 80 \%$ of lung cancer cases (1). Epidermal growth factor receptor $(E G F R)$ is frequently overexpressed in NSCLC and it is a promising target for individualized therapy (2). EGFR-targeted therapy with small-molecule tyrosine kinase inhibitors (TKIs), including gefitinib and erlotinib, has been approved for the treatment of advanced NSCLC (3). These inhibitors function by competitively binding at the adenosine triphosphate (ATP)-binding cleft of the receptor kinase domain, thereby blocking kinase activation and subsequent downstream signal transduction (3). However, TKIs are not effective in all NSCLC patients; an activating $E G F R$ mutation is a precondition for sensitivity (1).

Among the 29 types of common EGFR gene mutation, small in-frame deletions in exon 19 and heterozygous mutations of exon 21 (most commonly producing the L858R substitution), which is around the ATP-binding pocket, are the mutation hotspot, constituting $85-90 \%$ of all EGFR mutations $(3,4)$. The correlation between EGFR mutations and EGFR TKI sensitivity has been validated in several clinical trials and has been demonstrated to have potential prognostic value (5-7). Nevertheless, the majority of tumors become resistant to TKIs, mostly due to the incidence of a secondary T790M mutation, which is reported to negate the hypersensitivity of activating EGFR mutations (8).

Numerous methods for detecting EGFR mutation and thereby predicting the response to EGFR-targeting inhibitor therapy have emerged. Fluorescence in situ hybridization (FISH) is a strong predictor of survival benefit in patients with advanced NSCLC treated with EGFR inhibitors; however, it is labor intensive (9). Standard DNA-sequencing is able to detect tumor mutations within an abundance of $10-25 \%$, 
Table I. Primers used for plasmid construction.

\begin{tabular}{lll}
\hline Primers & Direction & \multicolumn{1}{c}{ Sequences } \\
\hline Exon20-WT & Forward & CTTTTCCTCCATGAGTACG \\
Exon20-WT & Reverse & AACTCTTGCTATCCCAGG \\
T790M & Forward & ACCGTGCAGCTCATCATGCAGCTCATGC \\
T790M & Reverse & ATGATGAGCTGCACGGTGGAGGTGAGGC \\
\hline
\end{tabular}

WT, wild-type.

depending on the quality of the tumors and genomic materials (10). The Scorpion ${ }^{\mathrm{TM}}$ amplification-refractory mutation system $\left(\right.$ ARMS $^{\mathrm{TM}}$ ) technique has a detection limit of $~ 1 \%$ (11). Methods based on quantitative polymerase chain reaction (qPCR) have been developed to evaluate mutation of the EGFR gene and are widely used clinically.

Recently, a new platform, named droplet digital polymerase chain reaction (ddPCR), has developed and is widely used for many clinical applications due to its unparalleled sensitivity and precision. ddPCR can quantify mutations in the EGFR gene in lung cancer at the single-molecule level, making it possible to detect samples with low mutation rates $(12,13)$. ddPCR uses a water-in-oil emulsion to generate up to 20,000 nanoliter-sized droplets that each contain no or some copies of a template and undergo separate end-point amplifications. A fluorescence detector is used to analyze these droplets, and a Poisson modeling equation is applied to measure the absolute number of copies of the target sequences.

In the present study, ARMS-qPCR and ddPCR methods were used to analyze plasmid samples and clinical samples, with the aim of comparing the performances of the two methods and exploring the feasibility of using ddPCR in the detection of samples with low mutation rates. The significance of and prospects for ddPCR in the detection of EGFR mutation, particularly the secondary EGFR T790M resistance mutation, were assessed. To the best of our knowledge, the present study is the first concerning the use of ddPCR for the detection of the EGFR T790M mutation and preliminarily illustrates the clinical significance of its early detection.

\section{Materials and methods}

Plasmid construction and plasmid sample preparation. Plasmid containing the EGFR T790M mutation was constructed as follows: Genomic DNA of HT-29 cells (ATCC ${ }^{\circledast}$ HTB-38 ${ }^{\mathrm{TM}}$; American Type Culture Collection, Manassas, VA, USA) was isolated using the TIANamp Blood DNA kit (cat. no. DP318-02; Tiangen Biotech Co., Ltd, Beijing, China). A DNA fragment containing the $E G F R$ gene was amplified from the genomic DNA of HT-29 cells using specific primers (Table I), and the PCR product was then ligated to pEASY-T1 vector (cat. no. CT101; TransGen Biotech Co., Ltd., Beijing, China). DNA sequencing was used to verify the accuracy of the fragment. The Fast Mutagenesis System was used to construct the EGFR T790M mutation with specific primers (Table I) following the manufacturer's instructions (cat. no. FM111; TransGen Biotech Co., Ltd.). The distinct T790M mutation was verified by sequencing. Different amounts of T790M mutant plasmids were mixed with wild-type EGFR plasmid to yield 6,30,60 and 300 copies of mutant molecules in 6,000 wild-type molecules, that is $0.1,0.5,1$ and $5 \%$ mutation rates, respectively. Haploid copy number dilutions were calculated based on the molecular weight of one normal haploid female genome equalling $3.275 \mathrm{pg}$ (12). Quantification was performed by the standard curve method using five standard dilutions, each in duplicate, of normal female genomic DNA ranging from 330 to $0.528 \mathrm{ng}(100,000$ to 160 haploid genome copies) per reaction.

Formalin-fixed paraffin embedded (FFPE) tumor samples from NSCLC patients. FFPE tumor samples obtained from patients between 2013 and 2014 were collected from the Affilated Hospital of Academy of Military Medical Sciences (Beijing, China). Written informed consent was obtained from all of the patients. The present study procedures were approved by the Institutional Review Board of the Affiliated Hospital of Academy of Military Medical Sciences. All tumor samples were routinely assessed by sectioning, hematoxylin and eosin staining, and visualization under a microscope to ensure that the tumor content was $\geq 50 \%$. If the tumor content was observed to be $<50 \%$, the samples were trimmed to satisfy the criteria. Genomic DNA was extracted using the QIAamp DNA FFPE Tissue kit (cat. no. 56404; Qiagen, Shanghai, China). Genomic DNA (gDNA) concentrations were determined by the measurement of optical density at $260 \mathrm{~nm}$ (OD260) using a Nanodrop 2000 spectrophotometer (Thermo Fisher Scientific, Wilmington, DE, USA) and the purity was evaluated by measurement of the OD260/OD280 ratio (all gDNA samples were between 1.92 and 1.97).

$q P C R$. The EGFR gene mutation statuses of the plasmid samples and 10 FFPE tumor samples were assessed using the Human EGFR Gene Mutations Detection kit (YZYMT-002-C; Wuhan YZY Medical Science and Technology Co., Ltd., Wuhan, China), to detect the 29 common EGFR mutations (G719A, G719S, G719C, S768I, T790M, L858R, L861Q, V769-D770insASV, H773-V774insH, D770-N771insG and the 19 common short in-frame deletions previously reported for $E G F R$ exon 19). The mutation assays were labeled with FAM, and each reaction mixture also contained a positive PCR control labeled with VIC. The templates were adjusted to a unified $10 \mathrm{ng} / \mu \mathrm{l}$ concentration, and the qualities of the templates were monitored and assessed by the reference system of the kit. qPCR was performed using a 7500 Real Time PCR system 
Table II. Detailed EGFR mutation detection results of the plasmid samples with 5-0.1\% mutation abundance.

\begin{tabular}{lccccc}
\hline Target & Sample (\%) & Copies & Positives & Negatives & Accepted droplets \\
\hline T790M & 5.0 & 398 & 245 & 13430 & 13675 \\
T790M & 1.0 & 57 & 35 & 13445 & 13480 \\
T790M & 0.5 & 24 & 14 & 12666 & 12680 \\
T790M & 0.5 & 24 & 4 & 13666 & 13681 \\
T790M & 0.1 & 7 & 3 & 13021 & 13025 \\
T790M & 0.1 & 5 & 0 & 13608 & 13949 \\
T790M & HT-29 & 0 & & 13608 \\
\hline
\end{tabular}

EGFR, epidermal growth factor receptor.

A

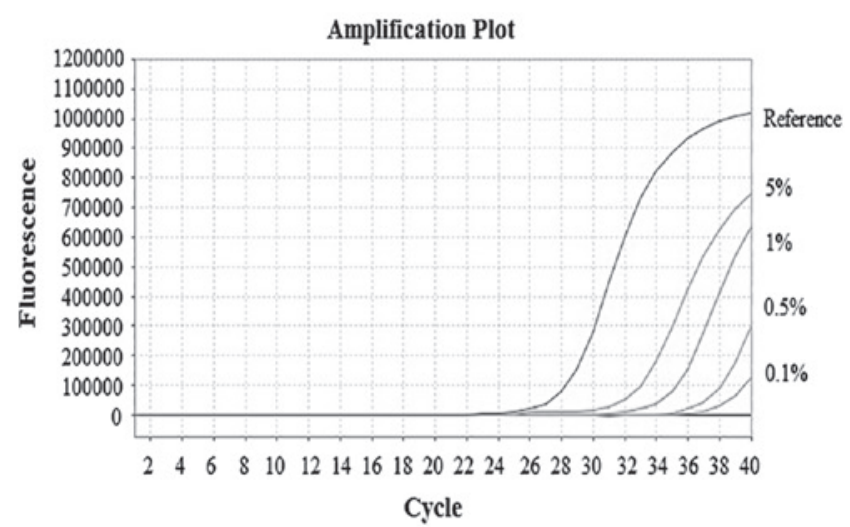

B

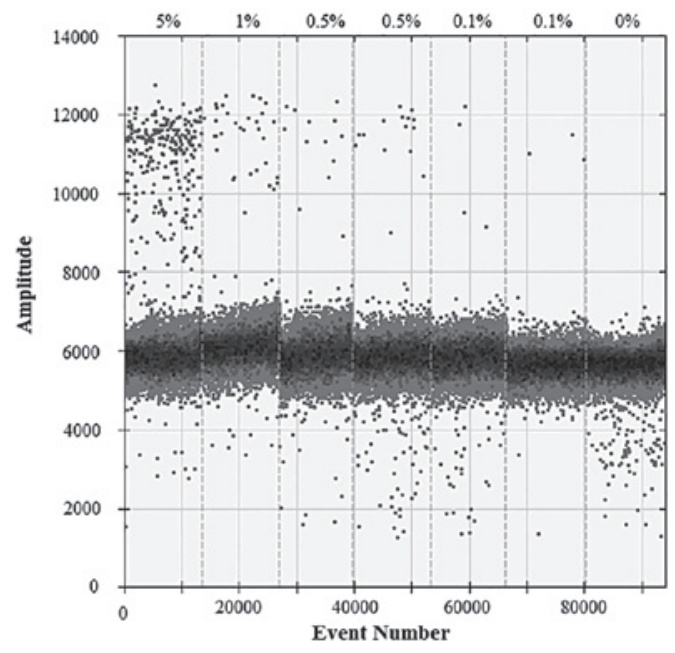

Figure 1 Quantitative performances of ARMS-qPCR and ddPCR for EGFR mutation analysis. EGFR mutation detection results of the plasmid samples with 5-0.1\% mutation rates by (A) ARMS-qPCR and (B) ddPCR methods. Mixtures of wild-type EGFR and T790M mutant plasmids present at fractional concentrations of 0.1 to $5 \%$ were analyzed with ARMS-qPCR and ddPCR. The total number of EGFR sequences in the sample was 6,000 per sample. EGFR, epidermal growth factor receptor; ARMS-qPCR, amplification refractory mutation system-based quantitative polymerase chain reaction; ddPCR, droplet digital polymerase chain reaction.

(Applied Biosystems Life Technologies, Shanghai, China). Thermocycling conditions were $95^{\circ} \mathrm{C}$ for $5 \mathrm{~min}$, followed by 40 cycles of $95^{\circ} \mathrm{C}$ for $15 \mathrm{sec}$ and $60^{\circ} \mathrm{C}$ for $1 \mathrm{~min}$. No template control (NTC) reactions were performed using water with no template, and in all cases, no amplification occurred.

ddPCR analysis. ddPCR was then performed to detect the $E G F R$ gene mutation statuses of the plasmid samples and the 10 FFPE tumor samples using a QX200 Droplet Digital PCR system (Bio-Rad Laboratories, Inc., Hercules, CA, USA). Reactions were performed in $25 \mu \mathrm{l}$ volumes using $12.5 \mu \mathrm{l}$ ddPCR 2X Master mix (Bio-Rad Laboratories, Inc.), $1.25 \mu 1$ 20X primer and TaqMan Probe mix (Applied Biosystems Life Technologies), $8.75 \mu \mathrm{l}$ nuclease-free water and $2.5 \mu \mathrm{l}$ template. Each sample was then loaded into the well of a droplet generator cartridge; $20 \mu 1$ sample was transferred into the middle wells of the cartridge, being careful to avoid bubbles and $70 \mu \mathrm{l}$ droplet generation oil (Bio-Rad Laboratories, Inc.) was added to the lower wells. The sample-containing cartridge was placed into the droplet generator to generate individual droplets. Once the process was complete, $40 \mu$ ldroplets were transferred into the wells of a 96-well PCR plate, sealed, and loaded into the thermal cycler. The following program was run: $95^{\circ} \mathrm{C}$ for $10 \mathrm{~min}$, followed by 40 cycles of $94^{\circ} \mathrm{C}$ for $30 \mathrm{sec}$ and $58^{\circ} \mathrm{C}$ for $1 \mathrm{~min}$, followed by $98^{\circ} \mathrm{C}$ for $10 \mathrm{~min}$, and holding at $4^{\circ} \mathrm{C}$. After PCR was complete, the sealed plate was loaded into the droplet reader for the detection of complete ddPCR reactions in individual droplets. The data was analyzed using QuantaSoft software (Bio-Rad Laboratories, Inc.) with the thresholds for detection set manually based on results from NTC wells containing water instead of DNA and the negative control using genomic DNA of HT-29 cells.

\section{Results}

EGFR mutation detection of the plasmid samples. Wild-type and EGFR T790M mutation plasmids were constructed and validated by sequencing. The plasmids were then quantified by ARMS-qPCR via the standard curve method using specific contents of HT29 gDNA. Copies of DNA $(n=6-300)$ from the EGFR T790M mutation plasmid were spiked into 6,000 copies of wild-type $E G F R$ plasmid to obtain plasmid samples with $0.1-5 \%$ mutation rates. Among the plasmid samples with $5,1,0.5$ and $0.1 \%$ T790M mutation rates, the ARMS-qPCR 
Table III. EGFR gene mutations in gDNA from samples of resected NSCLC tumors, as determined by the ARMS-qPCR and droplet digital PCR methods.

\begin{tabular}{lcclllll}
\hline $\begin{array}{l}\text { Sample } \\
\text { name }\end{array}$ & Gender & $\begin{array}{c}\text { Age, } \\
\text { years }\end{array}$ & $\begin{array}{c}\text { NSCLC } \\
\text { histology subtype }\end{array}$ & Stage & $\begin{array}{c}\text { ARMS- } \\
\text { qPCR }\end{array}$ & $\begin{array}{c}\text { ddPCR } \\
\text { (copy numbers) }\end{array}$ & Consistency \\
\hline N001 & F & 58 & Adenocarcinoma & IIB & WT & WT & Yes \\
N002 & M & 47 & Adenocarcinoma & IIIA & L858R & L858R (164) & Yes \\
N003 & F & 57 & Squamous cell & IIA & WT & WT & Yes \\
N004 & M & 68 & Adenocarcinoma & IIA & Del & Del (84) & Yes \\
N005 & M & 72 & Adenocarcinoma & IIB & WT & WT & Yes \\
N006 & F & 75 & Adenocarcinoma & IIIB & WT & T790M (7) & No \\
N007 & F & 58 & Adenocarcinoma & IIB & Del & Del (153) & Yes \\
N008 & M & 73 & Adenocarcinoma & IIB & WT & WT & Yes \\
N009 & M & 62 & Squamous cell & IIIA & WT & WT & Yes \\
N010 & F & 54 & Squamous cell & IA & WT & WT & Yes \\
\hline
\end{tabular}

EGFR, epidermal growth factor receptor; F, female; M, male; NSCLC, non-small cell lung cancer; ARMS-qPCR, amplification refractory mutation system-based quantitative polymerase chain reaction; ddPCR, droplet digital polymerase chain reaction; WT, wild-type; Del, deletion.
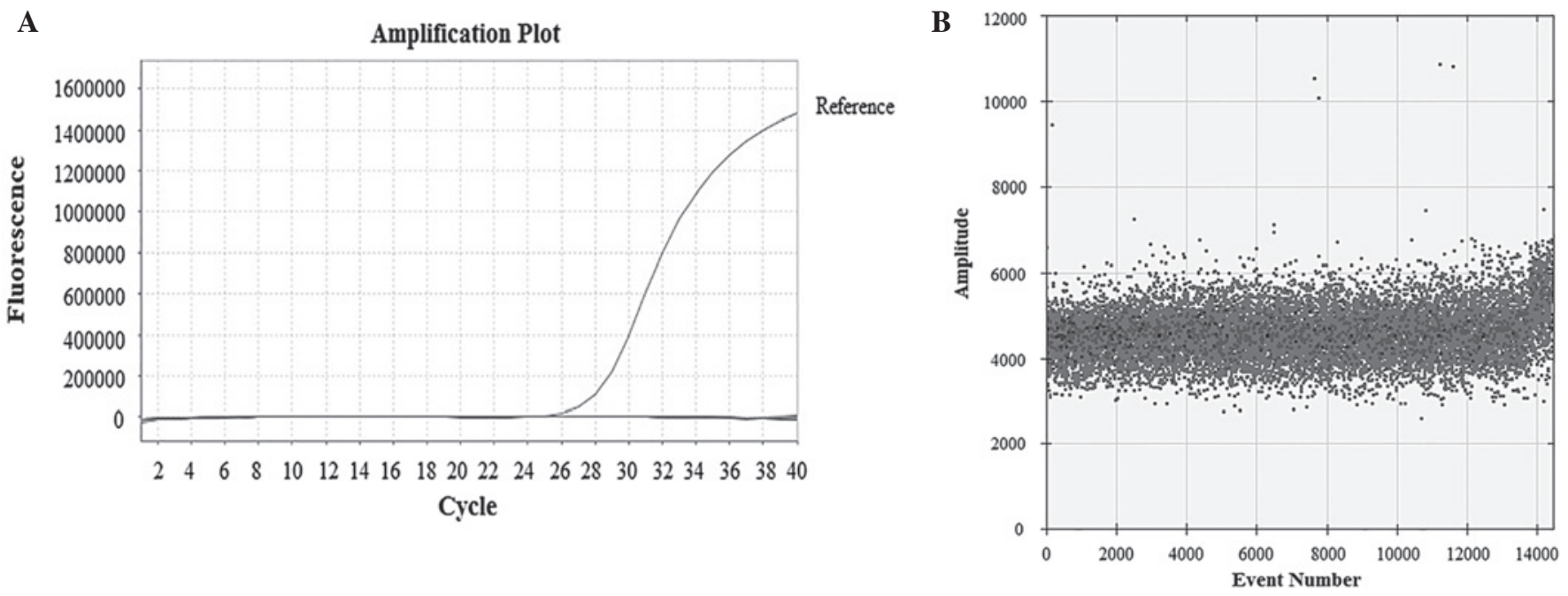

Figure 2. EGFR mutation detection results of the sample N006 by ARMS-qPCR and ddPCR methods. (A) The N006 sample was determined to be wild-type EGFR by qPCR. (B) The N006 sample showed the presence of seven mutated T790M alleles ( $0.1 \%$ mutant in the total genomic DNA from the tumor sample). EGFR, epidermal growth factor receptor; ARMS-qPCR, amplification refractory mutation system-based quantitative polymerase chain reaction; ddPCR, droplet digital polymerase chain reaction.

technology stably identified mutation in the plasmid samples with 5 and $1 \%$ mutation rates. The ddPCR method reliably detected mutation in the plasmid samples with all four mutation rates and calculated that the exact copy numbers (mean values) of the samples were $398,57,24$ and 6 , respectively; no mutation-positive copies were detected in the gDNA of HT-29 cells. These results are presented in Table II and Fig. 1.

EGFR mutation detection of the 10 FFPE tumor samples. Of the 10 FFPE tumor samples, the detection results of nine samples were consistent in the ARMS-qPCR and ddPCR methods. In two (20\%) of the 10 NSCLC tumor DNA samples tested, the presence of 84 and 153 molecules of an exon 19 deletion was identified among 6,000 genomic DNA copies (1.4\% and $2.55 \%$ ) in two samples of adenocarcinoma cell NSCLC tumor (stage IIA and IIB) obtained from a 68-year-old male and a 58-year-old female, respectively (Table III). In addition, the presence of L858R mutant EGFR alleles was identified in one (10\%) of the 10 samples examined, which showed 164 mutated alleles (2.73\%) among 6,000 genomic DNA copies (Table III). Notably, one sample named N006, which was considered as $E G F R$ wild-type when analyzed by ARMS-qPCR, was demonstrated to be an EGFR T790M mutation with seven copies of the mutation being present. The comparison results of the two methods are presented in Table III, and the detailed results of sample N006 using the ARMS-qPCR and ddPCR approaches are shown in Fig. 2.

\section{Discussion}

In this study, a series of plasmid samples with fractional $E G F R$ T790M mutation rates, varying from 5 to $0.1 \%$ in a background 
of 6,000 wild-type copies, were examined by conventional ARMS-qPCR and the newer platform ddPCR. It was found that plasmid samples with mutation rates as low as $1 \%$ were stably detected by ARMS-qPCR, while plasmid samples with mutation rates from 5 to $0.1 \%$ were reliably detected by ddPCR, with high precision and reproducibility, and the exact copy numbers of the mutant sequences were measured simultaneously. Subsequently, 10 FFPE samples were used to explore the performances of ARMS-qPCR and ddPCR in the detection of EGFR mutation. Of the 10 FFPE samples, detection results for nine samples were totally consistent by the two methods, while one sample was determined to be wild-type $E G F R$ by ARMS-qPCR but indicated to have an EGFR T790M mutation with seven copies of mutant molecules in a 6,000-copy wild-type background. These results illustrate that ddPCR can detect and quantify the presence of rare erlotinib/gefitinib-sensitizing EGFR mutations (0.12-2.73\%) at a much higher sensitivity than is possible with conventional ARMS-qPCR.

In the comparison of ARMS-qPCR and ddPCR in clinical application, the interpretation of ARMS-qPCR results depends on the threshold and quantification cycle $(\mathrm{Cq})$ value, and has a relatively high quality and concentration requirements for DNA templates (13). In this regard, ddPCR quantifies the sample by partitioning the sample and reaction components into thousands of reaction chambers, then counts the presence and absence of target molecules in each part of the sample following end-point PCR amplification $(3,13)$; therefore, it is independent of the $\mathrm{Cq}$ value and has a lower requirement for the DNA sample. Furthermore, partitioning the sample could decrease the amount of background DNA in the partition, thus enhancing the mutant concentration in each reaction, giving greater target amplification specificity and sensitivity $(3,13)$. Moreover, most clinical samples are FFPE tumor samples, in which DNA may be partially degraded (13); ddPCR is undoubtedly a good choice for these low quality DNA samples.

The results of the present study lend further support to the concept of EGFR mutation heterogeneity within lung tumors (14), in which EGFR-activating mutation exists to varying extents and even at very low abundances in early-stage lung tumors. Similar results were obtained in a earlier study involving the massively parallel sequencing of 22 lung adenocarcinoma specimens, which revealed that EGFR mutations can be very heterogeneous in a single tumor sample and that certain mutations were only present in $<10 \%$ of total sequences (15). There is little literature concerning EGFR mutation detection by digital PCR. In 2009, Yung et al reported the feasibility of using digital arrays in the detection of EGFR mutations in tumor tissues from patients with advanced metastatic NSCLC at a detection limit of $0.1 \%$ of the total number of 10,000 EGFR sequences (13). However, the significance of detecting low abundance mutations in tumor tissues was unclear at that time (13). In a later study, the detection and quantification of rare gefitinib/erlotinib-sensitizing EGFR mutations (0.02-9.26\% abundance) were reported in FFPE samples of early stage resectable lung tumors without an associated increase in gene copy number by digital array (3). The correlation between treatment response and low abundances of EGFR mutations was preliminary demon- strated in 2011; it was identified by direct DNA sequencing and ARMS approaches that lung cancer patients with low abundances $(\sim 1 \%)$ of EGFR mutations had a clearly longer median progression-free survival, higher objective response rate (ORR) and overall survival (OS) rate as compared with those of patient with wild-type $E G F R$, and the difference between patients with high and low abundances of $E G F R$ mutations was not found to be significant regarding ORR and OS (16). Further research is required to clarify the significance of lower EGFR mutation abundance $(<1 \%)$ detected by ddPCR.

In the two aforementioned papers concerning the detection of EGFR mutation by dPCR array, only in-frame deletion in exon 19 and L858R missense mutation in exon 21 were detected. Other gefitinib/erlotinib-sensitizing EGFR mutations, and more importantly, the T790M mutation, which can emerge during the treatment course with TKIs and then confer acquired drug resistance to the tumor cells, were not included. The EGFR mutant allele is heterogeneous, it may only present in a subset of tumor cells and can occur during cancer evolution; thus, the detection of the drug-resistant EGFR T790M mutation as early as possible before the acquired drug-resistance becomes clinically detectable is important, as it enables the physician to change the therapy promptly and give the patient the most effective treatment. In addition, ddPCR is a robust tool for achieving this objective, due to its high precision and sensitivity. Interestingly, the N006 sample in the present study, which was determined to be $E G F R$ wild-type by ARMS-qPCR, was demonstrated to have an EGFR T790M mutation by ddPCR.

In summary, this study indicated the advantages of ddPCR in low abundance EGFR mutation detection, particularly the TKI-resistant associated EGFR T790M mutation. It is expected that ddPCR may have good application prospects in molecular cancer diagnosis, for example in the study of phosphoinositide-3-kinase, catalytic subunit $\alpha$ (PIK3CA) (17), MET proto-oncogene (MET; hepatocyte growth factor) (18) and Kirsten rat sarcoma viral oncogene homolog (K-RAS) (19). Since ddPCR is able to detect rare mutants in a high background of wild-type sequences, it may play an increasing role in the field of gene mutation in peripheral blood circulating DNA (20) and circulating tumor cells $(21,22)$.

\section{References}

1. Bai H, Mao L, Wang HS, et al: Epidermal growth factor receptor mutations in plasma DNA samples predict tumor response in Chinese patients with stages IIIB to IV non-small-cell lung cancer. J Clin Oncol 27: 2653-2659, 2009.

2. Franklin WA, Veve R, Hirsch FR, Helfrich BA and Bunn PA Jr: Epidermal growth factor receptor family in lung cancer and premalignancy. Semin Oncol 29 (1 Suppl 4): 3-14, 2002.

3. Wang J, Ramakrishnan R, Tang Z, et al: Quantifying EGFR alterations in the lung cancer genome with nanofluidic digital PCR arrays. Clin Chem 56: 623-632, 2010.

4. Sharma SV, Bell DW, Settleman J and Haber DA: Epidermal growth factor receptor mutations in lung cancer. Nat Rev Cancer 7: 169-181, 2007.

5. Fukuoka M, Yano S, Giaccone G, et al: Multi-institutional randomized phase II trial of gefitinib for previously treated patients with advanced non-small-cell lung cancer (The IDEAL 1 Trial) [corrected]. J Clin Oncol 21: 2237-2246, 2003.

6. Kris MG, Natale RB, Herbst RS, et al: Efficacy of gefitinib, an inhibitor of the epidermal growth factor receptor tyrosine kinase, in symptomatic patients with non-small cell lung cancer: a randomized trial. JAMA 290: 2149-2158, 2003. 
7. Shepherd FA, Rodrigues Pereira J, Ciuleanu T, et al: Erlotinib in previously treated non-small-cell lung cancer. N Engl J Med 353: $123-132,2005$.

8. Yamada T, Azuma K, Muta E, et al: EGFR T790M mutation as a possible target for immunotherapy; identification of HLA-A*0201-restricted T cell epitopes derived from the EGFR T790M mutation. PLoS One 8: e78389, 2013.

9. Hirsch FR, Varella-Garcia M, Bunn PA Jr, et al: Molecular predictors of outcome with gefitinib in a phase III placebo-controlled study in advanced non-small-cell lung cancer. J Clin Oncol 24: 5034-5042, 2006.

10. Pao W and Ladanyi M: Epidermal growth factor receptor mutation testing in lung cancer: searching for the ideal method. Clin Cancer Res 13: 4954-4955, 2007.

11. Kimura H,Kasahara K, Kawaishi M, et al: Detection of epidermal growth factor receptor mutations in serum as a predictor of the response to gefitinib in patients with non-small-cell lung cancer. Clin Cancer Res 12: 3915-3921, 2006.

12. Whale AS, Huggett JF, Cowen S, et al: Comparison of microfluidic digital PCR and conventional quantitative PCR for measuring copy number variation. Nucleic Acids Res 40: e82, 2012.

13. Yung TK, Chan KC, Mok TS, Tong J, To KF and Lo YM: Single-molecule detection of epidermal growth factor receptor mutations in plasma by microfluidics digital PCR in non-small cell lung cancer patients. Clin Cancer Res 15: 2076-2084, 2009.

14. Nagai $Y$, Miyazawa $H$, Huqun, et al: Genetic heterogeneity of the epidermal growth factor receptor in non-small cell lung cancer cell lines revealed by a rapid and sensitive detection system, the peptide nucleic acid-locked nucleic acid PCR clamp. Cancer Res 65: 7276-7282, 2005.
15. Thomas RK, Nickerson E, Simons JF, et al: Sensitive mutation detection in heterogeneous cancer specimens by massively parallel picoliter reactor sequencing. Nat Med 12: 852-855, 2006.

16. Zhou Q, Zhang XC, Chen ZH, et al: Relative abundance of EGFR mutations predicts benefit from gefitinib treatment for advanced non-small-cell lung cancer. J Clin Oncol 29: 3316-3321, 2011.

17. Shigaki H, Baba Y, Watanabe M, et al: PIK3CA mutation is associated with a favorable prognosis among patients with curatively resected esophageal squamous cell carcinoma. Clin Cancer Res 19: 2451-2459, 2013.

18. Janjigian YY, Tang LH, Coit DG, et al: MET expression and amplification in patients with localized gastric cancer. Cancer Epidemiol Biomarkers Prev 20: 1021-1027, 2011.

19. Azuara D, Ginesta MM, Gausachs M, et al: Nanofluidic digital PCR for KRAS mutation detection and quantification in gastrointestinal cancer. Clin Chem 58: 1332-1341, 2012.

20. Gutiérrez C, Rodriguez J, Patiño-García A, García-Foncillas J, and Salgado J: KRAS mutational status analysis of peripheral blood isolated circulating tumor cells in metastatic colorectal patients. Oncol Lett 6: 1343-1345, 2013.

21. Marchetti A, Del Grammastro M, Felicioni L, et al: Assessment of EGFR mutations in circulating tumor cell preparations from NSCLC patients by next generation sequencing: Toward a real-time liquid biopsy for treatment. PLoS One 9: e103883, 2014.

22. Mostert B, Jiang Y, Sieuwerts AM, et al: KRAS and BRAF mutation status in circulating colorectal tumor cells and their correlation with primary and metastatic tumor tissue. Int J Cancer 133: 130-141, 2013. 